\title{
Team conflict management and $R \& D$ team effectiveness: The effects of task interdependence and team cooperation
}

\author{
Yen Hsu \\ The Graduate Institute of Design, Tatung University \\ No.40, Sec. 3,Zhongshan N. Rd., Taipei City 104,Taiwan, R.O.C. \\ Tel.: +886-2-2182-2928\#6731, Fax: +886-2-2586-7348 \\ E-mail address: erickshi@ms1.hinet.net
}

\begin{abstract}
Companies are dedicated to improving research and development $(R \& D)$ capacity to excel in market competition. Designers that share the same values and communicate and interact effectively are more likely to assist and cooperate with each other, making loyal contributions to the companies. This study explores the influence of task interdependence on engineering designers and examines cooperative conflict as a phenomenon. By conducting a questionnaire survey with 1000 consumer electronics manufacturers in Taiwan, we collected information regarding 339 design teams. Subsequently, we divided the teams based on various types of task interdependence before analyzing 28 cases of product development performed by these teams and investigating the cooperation and conflict between the team members. The results showed four types of task interdependence, namely, "Concretes", "efficiency", "concordance", and "specialization". Design teams featuring different types of task interdependence exhibit differing combinations in terms of the forms of team cooperation, team conflict, loyalty, and work values. Previous research has rarely addressed the conflict and cooperation of engineering designers within teams, and this study fills the research gap while offering specific recommendations for management practices.
\end{abstract}

KEYWORDS - Task interdependence, design team, team cooperation, team effectiveness, conflict

\section{INTRODUCTION}

"The next society will be a knowledge society. Knowledge will be its key resource, and knowledge workers will be the dominant group in its workforce" (Drucker, 2006; 2012). Bonuses and stock options no longer suffice as incentives to motivate designers, a type of knowledge workers, to fully demonstrate their abilities and contribute. Designers are loyal to their profession rather than the institutions that employ them. Therefore, managers should treat designers as partners rather than employees of an organization, avoiding making direct orders and commands. Drucker indicates that to cooperate with partners, one must first understand their values, objectives, and expectations. Previous studies on the relationship between team conflict and cooperation focused on issues such as cooperation within a group (Saavedra et al., 1993; Tjosvold, 1988), conflict management (Jehn, 1995), and team performance (Wageman, 1995). However, the majority of these studies explore the influence of interdependence on conflict or the influence of interdependence on cooperation, respectively, without investigating the interactions among the three factors. In addition, few studies have used engineering designers as the research subject. Nevertheless, the co-opetition theory developed based on the social interdependence theory introduces concepts such as cooperative conflict (Deutsch, 1973; Johnson \& Johnson, 1989; Tjosvold, 2008), which are worthy of further discussion. Hence, we use business design teams as the subjects to explore the relationships among the social interdependence, team conflict, team cooperation, work values, and loyalty of designers. The results can drive the theory development of conflict management and R\&D management and serve as a reference for engineering design managers.

\section{LITERATURE REVIEW}

\section{A. Task interdependence}

The interdependence among $R \& D$ team members can be considered as positive interdependence when members cooperate most efficiently to develop target market products. Task interdependence can be examined from two perspectives: team job design and team member perception. From the perspective of team job design, members must take initiatives to integrate and share raw materials, information, and professional knowledge with other members to achieve expected performance. With a higher level of interdependence and job difficulty, people have a stronger need to receive assistance from other people's work results (Van der Vegt\& Van de Vliert, 2002). From the perspective of team member perception, interdependence is the perception that team members must depend on other members to complete their tasks (Turner \& Lawrence, 1965). By examining interactions among R\&D team members, we believe positive team interdependence strengthens the sense of responsibility a person holds toward other team members. For example, people assist other team members in their work while completing their own work; people feel as miserable when they fail as when they cause other people to fail; teams with high interdependence strengthens individual people's achievement motivation and helping behavior (Matsui et. al., 1987). Therefore, we adopted the perspective of team member perception to explore the interdependence team members perceive when performing tasks, without discussing the factor of team job design.

Judging from the nature of work performed by engineering designers, team members who do not commit themselves to fulfilling the task affect team performance after a team task is initiated. High task interdependence can trigger a sense of responsibility in designers, motivating designers to improve personal performance while cooperating with and helping others, ultimately completing a task efficiently.

B. Team cooperation

Team cooperation can reflect the extent to which members value the shared goals and interests of a team. 
Conflicts arise when members care only about self-interests and personal traits or pay excessive attention to personal independence (Chatman \& Flynn, 2001). Team cooperation

(2003) stated that team cooperation is where employees can resolve conflict and formulate regulations when encountering multiple and complex problems. Schmidt and Kochan (1972) argue that both concepts involve incompatible member goals although the nature of the two concepts varies, the most significant difference being the perception of interdependence. Competition is where people achieve their goals without obstructing other people; in this situation, people do not perceive themselves as interdependent on other people. By contrast, conflict is where members are aware of job interdependence but the goals of the members are incompatible with each other. However, cooperation and conflict are two different concepts. Conflict is where individual people perceive that conflicting and incompatible expectations or uncompromisable desires exist within a team (Jehn\&Mannix, 2001). Therefore, the levels of conflict and cooperation within R\&D teams are correlated. Team members often compete against and cooperate with each other simultaneously. Members pursue common interests by sharing knowledge while using the shared knowledge as a competitive edge to exceed their peers. Competition affects the resource allocation in organizations and uneven resource allocation may also result in conflict (Tsai, 2002). Therefore, transforming team conflict to cooperative conflict enables members to adopt other people's views with an open heart and ultimately enhances team performance (Tjosvold, 1998). Competition and conflict are two similar concepts.

Ulrich and Eppinger (2015) suggest successful companies must organize R\&D team members effectively. $\mathrm{R} \& \mathrm{D}$ teams consist of individual designers and developers that are linked with each other. The link between members can be categorized into the following two types: linked by function or project. In functional organizations, the chief cooperation exists between members who perform similar functions, and in project organizations, the chief cooperation exists between members who execute the same projects. Ulrich and Eppinger (2015) further state that the two types may overlap, resulting in lightweight project matrix organizations and heavyweight project matrix organizations. By referencing the findings of Ulrich and Eppinger (2015), we explore, by examining the links of team cooperation, how engineering designers accomplish team goals with other people and how designers interact with other people.

\section{Team conflict, work values and loyalty}

Managed appropriately, conflict can yield benefits for organizations (Tjosvold, 2008). For example, Passos\& Caetano (2005) indicate, based on the group decision theory, that team conflict is beneficial for achieving critical thinking and ultimately benefits decision-making. From the perspective of cognition and learning, conflict can facilitate team decision-making processes, enhance the level of detail in decision selection (Putnam, 1994), and improve member cognition of the decision-making process (Simons \& Peterson, 2000). Therefore, Deutsch (1973) divides conflict into competitive and cooperative conflict. Specifically, cooperative conflict is where members emphasize a cooperative goal and allows teams to function and orients individual behavior toward a group, ultimately enabling members to accomplish team tasks (Hackman \& Oldham, 1976). Daily and Bishop

achieve the shared goal by assisting each other and communicating openly. Competitive conflict is where members perceive their goals as different from those of other members and thus believe they can accomplish their own goals only by sacrificing the goals of other people. Therefore, according to the coopetition theory, directing team conflict toward cooperative conflict can affect organizations positively. We conclude that the schools of conflict classification and coopetition management both agree conflict can affect performance positively under particular conditions or circumstances. In addition, $R \& D$ teams are characterized by positive interdependence, where conflict occurs while both parties work to achieve team goals (Deutsch, 1973; Tjosvold, 1991).

Robbins et al. (2003) argue work values lead people to develop biased perception of work itself and the real meaning of work, such as economic rewards, industriousness, loyalty, interpersonal relationship, social status, and self-realization. Work values act as standards based on which people offer thoughts and behave (Feather \&Rauter, 2004). Pisarski et al. (2008) state employees are less likely to come into conflicts within organizations when the ideas or values held by employees and teams match. Netemeyer et al. (1997) find conflicts are likely to arise when individual and organizational values contradict. Lockspeiser et al. (2008) show employees are likely to apply similar experience to resolve the problems they encounter when the values they hold are similar to those held by surrounding members; hence, a difference in opinion is unlikely to occur.

The majority of scholars regard organizational commitment as people's willingness to contribute effort and energy to an organization. Simultaneously, people identify with and are loyal to organizational values. In action, these people are willing to contribute to and work hard for the organization, hoping that they can continue to be part of the organization (Moliner et al., 2014). Therefore, identification with values is positively related to loyalty. When designers identify with the values of teams and organizations, the values of both parties are highly consistent. Therefore, designers are more loyal to an organization when they share the same values with the teams to which they belong. In other words, designers are more committed to an organization, willing to cooperate with the organization and actively participate in organizational tasks.

In summary, we can infer that conflicts are less likely to occur when R\&D team members share the same values or goals. When members share the same goals with an organization, they are loyal to the organization and are willing to work hard to achieve organizational goals. When members sharing the same goals gather, they play appropriate roles depending on their capabilities, pursing the shared goals wholeheartedly.

\section{RESEARCH METHODS}

We conducted a questionnaire survey on consumer electronics manufacturers operating in Taiwan to collect information regarding task interdependence within the engineering design or product development teams of these 
companies. The data were used to analyze the types of task interdependence existing in the corporate design teams. To design the questionnaire items, we referenced studies such as Kiggundu (1983). From the database, we randomly sampled 1000 engineering design or product development teams in consumer electronics manufacturers and sent out 1000 questionnaires. After constantly requesting replies, we obtained valid samples from 339 R\&D team leaders, for a response rate of $33.9 \%$. Conducted a reliability analysis of the data collected from the questionnaires and used principal components factoring, a factor analysis method, to categorize variables of task interdependence into the following five factor dimensions: "interaction and practicality", "communication quality", "cooperation and identification", "perceived value", and "goals and vision". Based on these six principal dimensions, we clustered the 339 design teams to classify characteristics of the team samples into various types.

A two-stage cluster analysis was performed in this study (Anderberg, 1973; Punj\& Stewart, 1983). First, we employed Ward's method, a cluster analysis method, to normalize the data when inputting the variables. Therefore, all variables shared the same unit. Subsequently, we used R2 and statistical prediction rule (SPR) and determined that the optimal number of clusters for 339 team samples was four $\left(R^{2}=.689\right.$, SPR $=.009)$. In the second stage, we employed the K-means method, a method of non-hierarchical cluster analysis, to reassign the samples to the most nearby cluster center based on the four cluster centers identified in the cluster analysis conducted in the first stage. Ultimately, we categorized the samples into four types. To understand various types of samples and investigate the differences between various strategy factor components and the characteristics of various types of samples, we used the five factor dimensions and variable items as independent variables and employed the four types as dependent variables to conduct one-way MANOVA. The results showed Wilks' lambda reached a level of significance of .01, indicating the four types differed significantly among the six dimensions. We further analyzed and named the four types based on their scores among the six factor dimensions, and the four types of task interdependence are Concretes, efficiency, concordance, and specialization (Table 1). Specifically, 69 samples were categorized as Concretes (20.4\%), 95 as efficiency (28.0\%), 61 as concordance (18.0\%), and 114 as specialization (33.6\%).

Table 1:Single factor ANOVA for all factors in each strategic types

\begin{tabular}{|l|l|l|l|l|l|l|}
\hline \multirow{2}{*}{ Dimensions } & \multicolumn{3}{|c|}{ Average factor score in eachdimension } & F & p \\
\cline { 2 - 5 } & Concretes & Efficiency & Concordance & Specialization & & \\
\hline $\begin{array}{l}\text { Interaction and } \\
\text { practicality }\end{array}$ & -.475 & .157 & .660 & -.447 & 14.154 & $.00^{*}$ \\
\hline $\begin{array}{l}\text { Communication } \\
\text { quality }\end{array}$ & -.344 & .598 & -.857 & -1.114 & 27.336 & $.00^{*}$ \\
\hline $\begin{array}{l}\text { Cooperation } \\
\text { and } \\
\text { identification }\end{array}$ & -.463 & -.257 & -.693 & .413 & 16.718 & $.00^{*}$ \\
\hline Perceived value & -.566 & .849 & -.513 & .097 & 23.458 & $.00^{*}$ \\
\hline $\begin{array}{l}\text { Goals and } \\
\text { vision }\end{array}$ & .576 & -.464 & -.748 & -.381 & 17.576 & $.00^{*}$ \\
\hline
\end{tabular}

Notes “*” represents reaching a significance level of 0.01 .
Using case analysis, we conducted in-depth interviews with R\&D team leaders by randomly sampling eight design teams from each of the four task interdependence types obtained from the questionnaire survey and analysis. In total, we interviewed 28R\&D team leaders and invited the interviewees to use their new products representative for the year's innovation as the basis for answering questions relating to the team cooperation, team conflict, loyalty, and work values within theirR\&D team. Table 2 shows the companies at which the interviewees' worked, the interviewee's job titles, and case company names.

Table 2:Types of task interdependence, company executives interviewed, and case products

\begin{tabular}{|c|c|c|c|c|c|}
\hline $\begin{array}{c}\text { Type of task } \\
\text { interdependence }\end{array}$ & $\begin{array}{l}\text { Company } \\
\text { name }\end{array}$ & $\begin{array}{l}\text { Department/personn } \\
\text { el interviewed }\end{array}$ & Interviewee & $\begin{array}{l}\text { Year's } \\
\text { innovation } \\
\text { project }\end{array}$ & $\begin{array}{l}\text { Case } \\
\text { No. }\end{array}$ \\
\hline \multirow{7}{*}{$\begin{array}{c}\text { A. } \\
\text { Concretes }\end{array}$} & Acer & $\begin{array}{l}\text { Assoc. Manager, } \\
\text { R\&D Depart. }\end{array}$ & Ku, H.C. & $\begin{array}{l}\text { Gaming } \\
\text { tablet }\end{array}$ & a1 \\
\hline & Benq & $\begin{array}{l}\text { Manager, Depart. of } \\
\text { Engineering Design }\end{array}$ & Liang, G.C. & $\begin{array}{l}\text { Digital } \\
\text { camera }\end{array}$ & $\mathrm{a} 2$ \\
\hline & ASUS & $\begin{array}{l}\text { Senior Manager, } \\
\text { Depart. of } \\
\text { Engineering Design }\end{array}$ & Mok, S.K. & $\begin{array}{l}\text { Smart } \\
\text { watch }\end{array}$ & a3 \\
\hline & Mitac & $\begin{array}{l}\text { Manager, R\&D } \\
\text { Depart. }\end{array}$ & Song, L.S. & $\begin{array}{l}\text { Smart } \\
\text { phone }\end{array}$ & $\mathrm{a} 4$ \\
\hline & Liteon & $\begin{array}{l}\text { Senior Manager, } \\
\text { Depart. of } \\
\text { Engineering Design }\end{array}$ & Pai, H.C. & $\begin{array}{l}\text { Mini } \\
\text { projector }\end{array}$ & a5 \\
\hline & Genius & $\begin{array}{l}\text { Manager, R\&D } \\
\text { Depart. }\end{array}$ & Ho, H.S. & $\begin{array}{l}\text { Photo card } \\
\text { reader }\end{array}$ & a6 \\
\hline & Tatung & $\begin{array}{l}\text { Manager, Products } \\
\text { R\&D Division }\end{array}$ & Pang, F. H. & Smart plug & a7 \\
\hline \multirow{7}{*}{$\begin{array}{c}\text { B. } \\
\text { Efficiency }\end{array}$} & Importek & $\begin{array}{l}\text { R\&D Director, R\&D } \\
\text { Depart. }\end{array}$ & Tsai, C.C. & $\begin{array}{l}\text { LED } \\
\text { projector }\end{array}$ & b1 \\
\hline & Moshi & $\begin{array}{l}\text { Assoc. Manager, } \\
\text { Depart. of Mechanic } \\
\text { Design }\end{array}$ & Hu, M.F. & $\begin{array}{l}\text { LED } \\
\text { reading } \\
\text { lamp }\end{array}$ & $\mathrm{b} 2$ \\
\hline & PQI & $\begin{array}{l}\text { Senior Manager, } \\
\text { R\&D Depart. }\end{array}$ & Lin, W.B. & $\begin{array}{l}\text { Heart-Rate } \\
\text { Smartwatch }\end{array}$ & b3 \\
\hline & Clevo & $\begin{array}{l}\text { Assoc. Manager, } \\
\text { R\&D Depart. }\end{array}$ & Chen, S. C. & $\begin{array}{l}\text { Network } \\
\text { video } \\
\text { recorder }\end{array}$ & $\mathrm{b} 4$ \\
\hline & Heran & $\begin{array}{l}\text { Manager, Depart. of } \\
\text { Innovation and R\&D }\end{array}$ & Hung, U.C. & $\begin{array}{l}\text { Bluetooth } \\
\mathrm{Hi}-\mathrm{Fi}\end{array}$ & b5 \\
\hline & Toongin & $\begin{array}{l}\text { Director, New } \\
\text { Products } \\
\text { Development } \\
\text { Division } \\
\end{array}$ & Tao, C.F. & $\begin{array}{l}\text { Wireless } \\
\mathrm{Hi}-\mathrm{Fi}\end{array}$ & b6 \\
\hline & Meiloon & $\begin{array}{l}\text { Manager, Depart. of } \\
\text { Mechanic Design }\end{array}$ & Wong, D.H. & $\begin{array}{l}\text { Digital } \\
\text { music } \\
\text { player }\end{array}$ & b7 \\
\hline \multirow{7}{*}{$\begin{array}{c}\text { C. } \\
\text { Concordance }\end{array}$} & Adata & $\begin{array}{l}\text { R\&D Chief, } \\
\text { Technology Officer }\end{array}$ & Liu, K.C. & LED bulb & $\mathrm{c} 1$ \\
\hline & \begin{tabular}{|l|} 
Transcen \\
d \\
\end{tabular} & $\begin{array}{l}\text { Manager, R\&D } \\
\text { Depart. }\end{array}$ & Wong, B.T. & \begin{tabular}{|l|}
$\begin{array}{l}\text { Car video } \\
\text { recorder }\end{array}$ \\
\end{tabular} & $\mathrm{c} 2$ \\
\hline & Behavior & $\begin{array}{l}\text { Manager, Depart. of } \\
\text { Engineering Design }\end{array}$ & Teng, W.S. & $\begin{array}{l}\text { Smart water } \\
\text { dispenser }\end{array}$ & c3 \\
\hline & Avision & $\begin{array}{l}\text { Manager, Depart. of } \\
\text { Engineering Design }\end{array}$ & Jin, S.G. & $\begin{array}{l}\begin{array}{l}\text { Portable } \\
\text { scanner }\end{array} \\
\end{array}$ & $\mathrm{c} 4$ \\
\hline & CviLux & $\begin{array}{l}\text { Senior Manager, } \\
\text { Depart. of } \\
\text { Engineering Design }\end{array}$ & Ching, C.C. & $\begin{array}{l}\text { Carry } \\
\text { power }\end{array}$ & c5 \\
\hline & S\&T & $\begin{array}{l}\text { R\&D Chief, } \\
\text { Technology Officer }\end{array}$ & Yang, C.M. & $\begin{array}{l}\text { Smart air } \\
\text { purifier }\end{array}$ & c6 \\
\hline & Mitac & $\begin{array}{l}\text { Manager, Depart. of } \\
\text { Product R\&D }\end{array}$ & Ko, K.Z. & $\begin{array}{l}\text { Bike } \\
\text { navigation }\end{array}$ & c7 \\
\hline \multirow{4}{*}{$\begin{array}{c}\text { D. } \\
\text { Specialization }\end{array}$} & Portoti & $\begin{array}{l}\text { Vice } \\
\text { President,Depart. of } \\
\text { R\&D }\end{array}$ & Tung, S.L. & $\begin{array}{l}\text { Mosquito } \\
\text { trap }\end{array}$ & d1 \\
\hline & Dlink & $\begin{array}{l}\text { Assoc. Manager, } \\
\text { Depart.of R\&D }\end{array}$ & Kang, E.W. & IP camera & $\mathrm{d} 2$ \\
\hline & $\begin{array}{l}\text { Thermalt } \\
\text { ake }\end{array}$ & $\begin{array}{l}\text { Senior Manager, } \\
\text { Depart. of } \\
\text { Engineering Design }\end{array}$ & Lai, C.H. & $\begin{array}{l}\text { Gaming } \\
\text { headset }\end{array}$ & $\mathrm{d} 3$ \\
\hline & Heran & Assoc. Manager, & Chong, & Inverter air & $\mathrm{d} 4$ \\
\hline
\end{tabular}




\begin{tabular}{|l|l|l|l|l|c|}
\hline & & $\begin{array}{l}\text { Depart. of New } \\
\text { Product Innovation }\end{array}$ & M.C. & conditioner & \\
\cline { 2 - 5 } & Apacer & $\begin{array}{l}\text { Manager, Depart. of } \\
\text { R\&D Design }\end{array}$ & Fang, H.S. & Flash drive & d5 \\
\cline { 2 - 5 } ZyXEL & $\begin{array}{l}\text { Manager, Depart.of } \\
\text { R\&D }\end{array}$ & Chien, L.F. & $\begin{array}{l}\text { Wi-Fi } \\
\text { router }\end{array}$ & d6 \\
\cline { 2 - 5 } & $\begin{array}{l}\text { Silicon } \\
\text { Power }\end{array}$ & $\begin{array}{l}\text { Senior Manager, } \\
\text { Depart. of Mechanic } \\
\text { Design }\end{array}$ & Ma, C.M. & $\begin{array}{l}\text { Gaming } \\
\text { keyboard }\end{array}$ & d7 \\
\hline
\end{tabular}

\section{FINDINGS}

Table 3 shows the relationships among team cooperation, team conflict, loyalty, and work values in design teams featuring differing types of task interdependence. Specifically, the most common type of team cooperation was function-based and lightweight matrix-based links (8\& 9 cases for T1 and T3, respectively), followed by project-based and heavyweight matrix-based links ( $5 \& 6$ cases for T2 and $\mathrm{T} 4$, respectively).

Table 3: A comparison of team cooperation, team conflict, loyalty, and work values among teams featuring various types of task interdependence

\begin{tabular}{|c|c|c|c|c|c|c|c|c|c|c|c|c|c|c|c|c|c|c|c|c|c|}
\hline \multirow[t]{2}{*}{ Type } & \multirow{2}{*}{$\begin{array}{c}\text { Cas } \\
\mathrm{e}\end{array}$} & \multicolumn{4}{|c|}{$\begin{array}{c}\text { Team } \\
\text { cooperati } \\
\text { on }\end{array}$} & \multicolumn{6}{|c|}{$\begin{array}{c}\text { Team } \\
\text { conflict }\end{array}$} & \multicolumn{4}{|c|}{ Loyalty } & \multicolumn{6}{|c|}{ Work values } \\
\hline & & $\mathrm{T}$ & $\mathrm{T}$ & $\mathrm{T}$ & $\begin{array}{l}\mathrm{T} \\
4\end{array}$ & $\mathrm{~F}$ & $\begin{array}{l}\mathrm{F} \\
2\end{array}$ & $\begin{array}{l}F \\
3\end{array}$ & $\begin{array}{l}\mathrm{F} \\
4\end{array}$ & \begin{tabular}{|l}
$\mathrm{F}$ \\
5
\end{tabular} & $\begin{array}{l}F \\
6\end{array}$ & $\begin{array}{l}\mathrm{L} \\
1\end{array}$ & $\begin{array}{l}\mathrm{L} \\
2\end{array}$ & $\begin{array}{l}\mathrm{L} \\
3\end{array}$ & & & $\begin{array}{l}V \\
2\end{array}$ & & & & \\
\hline \multirow{8}{*}{ Concre } & 1 & & $\mathrm{X}$ & & & & & & $\underline{x}$ & & & & & & $x$ & & $\mathrm{x}$ & & & & \\
\hline & 2 & & $\mathrm{X}$ & & & & & & $\mathrm{x}$ & & & & & & $\mathrm{x}$ & $\mathrm{X}$ & & & & & \\
\hline & a3 & & & & $\mathrm{X}$ & & & $\mathrm{X}$ & & & & & & $\mathrm{x}$ & & $\mathrm{X}$ & & & & & \\
\hline & $\mathrm{a} 4$ & & $\mathrm{X}$ & & & & & $\mathrm{x}$ & & & & $\mathrm{x}$ & & & & & $\mathrm{x}$ & & & & \\
\hline & a5 & & & & $\mathrm{X}$ & & & $\mathrm{x}$ & & & & & & & $\mathrm{x}$ & $\mathrm{x}$ & & & & & \\
\hline & a6 & & $X$ & & & & & & $\underline{x}$ & & & & & & $\mathrm{x}$ & $\mathrm{x}$ & & & & & \\
\hline & a7 & $x$ & & & & & & & $\mathrm{x}$ & & & & & & $\mathrm{x}$ & & & & & & \\
\hline & Sub & 1 & 4 & 0 & 2 & 0 & 0 & 3 & 4 & 0 & 0 & 1 & 0 & 1 & 5 & 4 & 2 & $\underline{0}$ & 0 & $\begin{array}{ll}0 \\
0\end{array}$ & \\
\hline \multirow{8}{*}{$\begin{array}{c}\text { Efficie } \\
\text { ncy }\end{array}$} & b1 & & & $\underline{x}$ & & & & & & $\underline{x}$ & & & $x$ & & & & & & & & \\
\hline & b2 & & & & & & & & & $\underline{x}$ & & & & $\underline{x}$ & & & & & & $\underline{x}$ & \\
\hline & b3 & & & $\mathrm{X}$ & & & & & & $x$ & & & $\mathrm{x}$ & & & & & & & & \\
\hline & \begin{tabular}{|l|} 
b4 \\
\end{tabular} & $X$ & & & & & & & & $\mathrm{x}$ & & & $\mathrm{x}$ & & & & & & & & \\
\hline & b5 & & & $\underline{X}$ & & & & & & $\mathrm{x}$ & & & $x$ & & & & & & & & \\
\hline & $\mathrm{b}$ & & & $\mathrm{X}$ & & & & & & & $\mathrm{x}$ & & $\underline{x}$ & & & & & & & $\underline{x}$ & \\
\hline & b7 & & & $\mathrm{X}$ & & & & & & & $\mathrm{x}$ & & 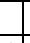 & & $\mathrm{x}$ & & & & & & \\
\hline & Sub & 2 & 0 & 5 & 0 & 0 & 0 & 0 & 0 & 5 & 21 & 0 & 5 & 1 & 1 & 0 & 0 & 0 & 0 & & 3 \\
\hline \multirow{8}{*}{$\begin{array}{l}\text { Concor } \\
\text { dance }\end{array}$} & c1 & & & X & & $\mathrm{X}$ & & & & & & $\mathrm{x}$ & & & & & & $\mathrm{X}$ & & & \\
\hline & $\mathrm{c} 2$ & & $x$ & & & & & $\mathrm{x}$ & & & & $\mathrm{x}$ & & & & & & $\mathrm{x}$ & & & \\
\hline & c. & & & & $\mathrm{X}$ & & $\mathrm{X}$ & & & & & & & $\mathrm{x}$ & & & & $\mathrm{x}$ & & & \\
\hline & $\mathrm{c} 4$ & & & $\underline{x}$ & & $\mathrm{x}$ & & & & & & $\underline{x}$ & & & & & & & $\underline{x}$ & & \\
\hline & c5 & & & & $\mathrm{X}$ & & $\underline{x}$ & & & & & $\underline{x}$ & & & & & & & $x$ & & \\
\hline & c6 & & & $\underline{X}$ & & $\underline{X}$ & & & & & & $\underline{x}$ & & & & & & $\underline{x}$ & & & \\
\hline & $\begin{array}{l}\text { c7 } \\
\end{array}$ & & & & $\mathrm{X}$ & & $\mathrm{x}$ & & & & & & & & $\mathbf{x}$ & & & & $\bar{x}$ & & \\
\hline & Sub & 0 & 1 & 3 & 3 & 3 & 3 & 1 & 0 & 0 & 0 & 5 & 0 & 1 & 1 & 0 & $\underline{0}$ & 4 & 3 & & ( \\
\hline \multirow{9}{*}{$\begin{array}{l}\text { Special } \\
\text { ization }\end{array}$} & d1 & $\mathrm{X}$ & & & & & & & & & $\underline{x}$ & & & $\underline{x}$ & & & $\underline{x}$ & & & & \\
\hline & $\mathrm{d} 2$ & & & $\underline{x}$ & & & & & & & $\underline{X}$ & & & $\underline{x}$ & & $\mathrm{X}$ & & & & & \\
\hline & $\mathrm{d} 3$ & & & & $\mathrm{X}$ & & $\underline{x}$ & & & & & & & & $\underline{x}$ & $\mathrm{x}$ & & & & & \\
\hline & $\mathrm{d} 4$ & $\mathrm{X}$ & & & & & & & & & $\underline{x}$ & & & $\underline{\underline{x}}$ & & & $\underline{x}$ & & & & \\
\hline & $\mathrm{d} 5$ & $\underline{X}$ & & & & & & & & & $\mathrm{x}$ & & & $\underline{X}$ & & $\mathrm{x}$ & & & & & \\
\hline & d6 & & & & & & & & & $\underline{x}$ & & & & $\underline{x}$ & & $\mathrm{X}$ & & & & & \\
\hline & $\mathrm{d} 7$ & $\underline{X}$ & & & & & & & & & $\mathrm{x}$ & & $\underline{x}$ & & & 4 & $\underline{x}$ & & & & \\
\hline & Sub & 5 & 0 & 1 & 1 & 0 & 1 & 0 & 0 & 1 & 5 & 0 & 1 & 5 & 1 & 4 & $\frac{3}{-2}$ & 0 & 0 & 0 & 0 \\
\hline & Tot & 8 & 5 & 9 & 6 & 3 & 4 & 4 & 4 & 6 & 7 & 6 & $\underline{6}$ & 8 & 8 & 8 & 5 & 4 & & $4 \mid 2$ & 2 \\
\hline
\end{tabular}

Notes: "x" indicates a case was assigned to a specific category.

Types of links in team cooperation: Function (T1), project (T2), lightweight matrix (T3), heavyweight matrix (T4)

Team conflict: Cognitive (F1), goal (F2), interpersonal (F3), emotion (F4), process (F5), methodological(F6)

Loyalty: Identification with organizations (L1), proactivity (L2), cooperation and compliance (L3), devotion to duty (L4) Work values: Social glory (V1), remuneration and benefits (V2), autonomy (V3), sense of achievement (V4), interpersonal relationship (V5), work environment (V6)

Design teams featuring Concretes as the type of task interdependence were characterized by project-based links as the primary form of team cooperation (T2). Design teams featuring efficiency as the type of task interdependence were characterized by lightweight matrix-based links as the primary form of team cooperation (T3). Design teams featuring concordance as the type of task interdependence were characterized by lightweight matrix-based and heavyweight matrix-based links as the forms of team cooperation (T3; T4). Design teams featuring specialization as the type of task interdependence were characterized by function-based links as the primary form of team cooperation (T1).

The most frequently observed type of team conflict was procedural conflict ( 6 cases for F5; 7 cases for F6), and the frequency of task conflict and relationship conflict was distributed evenly. In design teams featuring Concretes as the type of task interdependence, the most frequently observed type of team conflict was relationship conflict, such as conflicts at the interpersonal and emotion levels (F3; F4). The primary form of team conflict observed in design teams featuring efficiency as the type of task interdependence was procedural conflict (F5). In design teams featuring concordance as the type of task interdependence, the primary type of team conflict was cognitive conflict (F1). In design teams featuring specialization as the type of task interdependence, the primary form of team conflict was methodological conflict (F6).

The most commonly observed type of loyalty was cooperation and compliance ( 8 cases for L3), followed by devotion to duty ( 8 cases for L4), indicating these were the two types of loyalty most frequently observed in the R\&D team members examined in this study. In design teams featuring Concretes as the type of task interdependence, loyalty was primarily characterized by devotion to duty (L4). In design teams featuring efficiency as the type of task interdependence, loyalty was primarily characterized by proactivity (L2). In design teams featuring concordance as the type of task interdependence, loyalty was primarily characterized by identification with organizations (L1). In design teams featuring specialization as the type of task interdependence, loyalty was characterized by cooperation and compliance (L3).

The most frequently observed work value was social glory (8 cases for V1), indicating social glory was the work value most commonly seen in the design teams. Observingthe individual cases showed that design teams featuring Concretes as the type of task interdependence primarily satisfied their team members with the work values of social glory (V1). Design teams featuring efficiency as the type of task interdependence primarily satisfied their team members with the work values of interpersonal relationship and work environment (V5; V6). Design teams featuring concordance as the type of task interdependence primarily satisfied their team members with the work values of autonomy (V3). Design teams featuring specialization as the type of task 
interdependence primarily satisfied their team members with the work values of social glory and compensation and benefits (V1; V2).

\section{DISCUSSIONS}

Amid market competition, design teams in consumer electronics manufacturers operating in Chinese-speaking countries can be divided into the following four categories based on the type of task interdependence: Concretes, efficiency, concordance, and specialization. After examining cases featuring various types of task interdependence, we identified the following four types of links in the team cooperation of R\&D team members: function-, project-, lightweight matrix-, and heavyweight matrix-based links. During the process of team cooperation, six types of task conflicts arise, namely, cognitive conflict (at the cognitive and goal levels), relationship conflict (at the interpersonal and emotion levels), and procedural conflict (at the process and methodological levels). Teams featuring function- and lightweight matrix-based links in team cooperation were characterized by procedural conflict as the primary type of team conflict; teams featuring project-based links exhibited relationship conflict, and teams featuring heavyweight matrix-based links were characterized by cognitive conflict.

Based on the type of team loyalty, the case design teams can be divided into the following four categories: identification with organizations, proactivity, cooperation and compliance, and devotion to duty. In addition, the teams were characterized by behavioral loyalty, such as cooperation and compliance and devotion to duty. Regarding work values, we identified six types of work values: extrinsic values (social glory and remuneration and benefits), intrinsic values (autonomy and sense of achievement), and additional values (interpersonal relationship and work environment). Specifically, two types of team loyalty, cooperation and compliance, and devotion to duty, were primarily prompted by extrinsic work values. By contrast, identification with organizations as another type of team loyalty was prompted by intrinsic values, and proactivity was prompted by additional values.

Design teams featuring different types of task interdependence exhibited various combinations in terms of the forms of team cooperation, team conflict, loyalty, and work values:1) Design teams featuring Concretes as the type of task interdependence were characterized by project-based links (T2) as the form of team cooperation; interpersonal (F3) and emotional (F4) conflict as the primary type of team conflict; devotion to duty (L4) as the form of team member loyalty; and social glory (V1) as the primary type of work values; 2) Design teams featuring efficiency as the type of task interdependence were characterized by lightweight matrix-based links (T3) as the form of team cooperation; procedural conflict (F5) as the primary type of team conflict; proactivity (L2) as the form of team member loyalty; and interpersonal relationship (V5) and work environment (V6) as the primary types of work values; 3) Design teams featuring concordance as the type of task interdependence were characterized by lightweight matrix-based links (T3) and heavyweight matrix-based links (T4) as the primary forms of team cooperation; cognitive conflict (F1) as the primary type of team conflict; identification with organizations (L1) as the form of team member loyalty; and autonomy (V3) as the primary type of work values; 4) Design teams featuring specialization as the type of task interdependence were characterized by function-based links (T1) as the primary form of team cooperation; methodological conflict (F6) as the primary type of team conflict; cooperation and compliance (L3) as the form of team member loyalty; and social glory (V1) and compensation and benefits (V2) as the primary types of work values.

Companies are confronted with multiple challenges in the rapidly evolving consumer electronics market. Launching new products swiftly and relying on teamwork to perform product development is often an effective coping strategy. Simultaneously, team organization must constantly innovate for businesses to remain competitive and survive in the industry (Huang et al., 2012; Bart, 2012). With the diversity of innovative team members increasing in Chinese companies, communication between members from differing cultures is common. Conflicts are inevitable during the process of communication, and using conflict properly can drive group development (De Dreu, 1997; Jehnet al., 2013). However, handling conflict inappropriately can hinder organizational operations. Therefore, while companies emphasize the importance of cooperation in design teams, building effective communication channels is a vital factor for sustaining proper organizational operation (Mohrmanet al., 1995).

\section{CONCLUSIONS}

Previous studies have shown that when conflict arises in communication, the creative results of team brainstorming fall short of the output of individual thinking (Goldschmidt, 1995; Pirola-Merlo \& Mann, 2004; Caputo, 2013). Therefore, proper conflict management can resolve conflict and direct the power of conflict to the advantage of organizations (Rahim \&Bonoma, 1979; Segi, 2013). During the process of case studying, we found the likelihood of organizational conflict decreased when designers and teams shared the same or similar values. In this situation, designers had amicable relationships with each other, and the designers were more loyal to the team organizations to which they belonged. Subsequently, team cooperation improved. Hence, we recommend business owners to value the consistency of the values held by designers, team members, and corporate organizations and focus on enhancing designers' identification with team organizations. For example, managers should listen to and communicate with designers more frequently. In addition, directors of design departments should provide a list of traits they prefer in designers to the human resources department when hiring new designers. By so doing, hiring teams can identify designers most suitable for the organizations.

In view of team conflict, team relationship, and loyalty being the antecedents of team cooperation, we recommend business executives pay more attention to the communication between and mentality of R\&D team members. Team conflict and team relationship are interpersonal problems, both of which do not result from short-term issues. Therefore, department heads should always pay more attention to the communication between designers to prevent team conflict from occurring. Department heads can use appropriate opportunities to encourage communication between designers and improve relationship quality. The team loyalty of engineering designers is often overlooked by companies. 
Designers staying with a company are not necessarily loyal to the company. Hence, companies should pay attention to the mentality of designers. Instead of merely ensuring tasks are completed, managers should understand the mentality under which designers complete tasks. To motivate designers to cooperate with teams, managers should improve the consistency between the values held by designers, teams, and organizations, while reducing organizational conflict, improving relationship quality, and enhancing the loyalty of designers to companies.

\section{ACKNOWLEDGEMENTS}

The author would like to thank the anonymous reviewers for valuable comments and suggestions during the review process. This study was supported by the Tatung University (TTU) as well asMinistry of Science and Technology (MOST), Taiwan, R.O.C. He also would like to thank the graduate students for assistance data collection in the study process.

\section{REFERENCES}

[1] Anderberg, M. R. (1973). Cluster Analysis for Application. New York: Academic Press.

[2] Bart, C. F. C. A. (2012). Innovation and structure - the perfect corporate match! The Canadian Manager, 37(2), 21-22,24.

[3] Caputo, A. (2013). A literature review of cognitive biases in negotiation processes. International Journal of Conflict Management, 24(4), 374-398.

[4] Chatman, J. A., \& Flynn, F. J. (2001). The influence of demographic heterogeneity on the emergence and consequences of cooperative norms in work teams. Academy of Management Journal, 44(5), 956-974.

[5] Chen, S. C. (2016, July 3). [Associate Manager, R\&D Department, Clevo, Interview by author].

[6] Chien, L. F. (2016, September 30). [Manager, Department of R\&D, ZyXEL, Interview by author]

[7] Ching, C. C. (2016, September 21). [Senior Manager, Department of Engineering Design, CviLux, Interview by author].

[8] Chong, M. C. (2016, August 11). [Associate Manager, Department of New Product Innovation, Heran, Interview by author].

[9] Daily, B. F., \& Bishop, J. W. (2003). TQM workforce factors and employee involvement: the pivotal role of teamwork. Journal of Managerial Issues, 393-412.

[10] De Dreu, C. K., Evers, A., Beersma, B., Kluwer, E. S., \&Nauta, A. (2001). A theory $\square$ based measure of conflict management strategies in the workplace. Journal of Organizational Behavior, 22(6), 645-668.

[11] De Dreu, C. K. W., \& Van de Vliert, E. (1997). Using Conflict in Organizations: SAGE Publications.

[12] Deutsch, E. (1973). AdvaitaVed_nta: A Philosophical Reconstruction: University of Hawaii Press.

[13] Deutsch, M. (1973). The resolution of conflict. New Haven, CT: Yale.

[14] Deutsch, M. (1985). Distributive justice: A social-psychological perspective.

[15] Drucker, P. (2012). Managing in the Next Society: Taylor \& Francis.

[16] Drucker, P. F. (2006). Classic Drucker: Essential Wisdom of Peter Drucker from the Pages of Harvard Business Review: Harvard Business Review Book.

[17] Fang, H. S. (2016, August 7). [Manager, Department of R\&D Design, Apacer, Interview by author].

[18] Gil, F., Alcover, C.-M., Peiró, J.-M., Margarida Passos, A., \& Caetano, A. (2005). Exploring the effects of intragroup conflict and past performance feedback on team effectiveness. Journal of managerial psychology, 20(3/4), 231-244.

[19] Hackman, J. R., \& Oldham, G. R. (1976). Motivation through the design of work: Test of a theory. Organizational behavior and human performance, 16(2), 250-279.

[20] Ho, H. S. (2016, July 28). [Manager, R\&D Department, Genius, Interview by author].

[21] Hu, M. F. (2016, July 23). [Associate Manager, Department of Mechanic Design, Moshi, Interview by author].

[22] Huang, H.-C., Lai, M.-C., Kao, M.-C., \& Chen, Y.-C. (2012). Target Costing, Business Model Innovation, and Firm Performance: An Empirical Analysis of Chinese Firms. Canadian Journal of Administrative Sciences, 29(4), 322

[23] Hung, U. C. (2016, July 10). [Manager, Department of Innovation and
$R \& D$, Heran, Interview by author].

[24] Jehn, K., Rispens, S., Jonsen, K., \& Greer, L. (2013). Conflict contagion: a temporal perspective on the development of conflict within teams. International Journal of Conflict Management, 24(4), 352-373.

[25] Jehn, K. A. (1995). A multimethod examination of the benefits and detriments of intragroup conflict. Administrative science quarterly, 40(2), 256-282.

[26] Jehn, K. A. (1997). A qualitative analysis of conflict types and dimensions in organizational groups. Administrative science quarterly, 42(3), 530-557.

[27] Jehn, K. A., \&Mannix, E. A. (2001). The dynamic nature of conflict: A longitudinal study of intragroup conflict and group performance. Academy of Management Journal, 44(2), 238-251.

[28] Jin, S. G. (2016, July 16). [Manager, Department of Engineering Design, Avision, Interview by author].

[29] Johnson, D. W., \& Johnson, R. T. (1989). Cooperation and competition: Theory and research: Interaction Book Company.

[30] Kang, E. W. (2016, September 25). [Associate Manager, Department of $\mathrm{R} \& \mathrm{D}$, Dlink, Interview by author]

[31] Kiggundu, M. N. (1981). Task interdependence and the theory of job design. Academy of management Review, 6(3), 499-508.

[32] Kiggundu, M. N. (1983). Task interdependence and job design: Test of a theory. Organizational behavior and human performance, 31(2), $145-172$

[33] Ko, K. Z. (2016, September 11). [Manager, Department of Communications Product R\&D, Mitac, Interview by author].

[34] Ku, H. C. (2016, July 1). [Associate Manager, R\&D Department, Acer, Interview by author]

[35] Lai, C. H. (2016, September 7). [Senior Manager, Department of Engineering Design, Thermaltake, Interview by author].

[36] Liang, G. C. (2016, August 18). [Manager, Department of Engineering Design, Benq, Interview by author]

[37] Lin, W. B. (2016, July 20). [Senior Manager, R\&D Department, PQI, Interview by author]

[38] Liu, K. C. (2016, July 24). [R\&D Chief, Technology Officer, Adata, Interview by author]

[39] Lockspeiser, T. M., O'Sullivan, P., Teherani, A., \& Muller, J. (2008). Understanding the experience of being taught by peers: the value of social and cognitive congruence. Advances in Health Sciences Education, 13(3), 361-372.

[40] Ma, C. M. (2016, September 9). [Senior Manager, Department of Mechanic Design, Silicon Power, Interview by author].

[41] Matsui, T., Kakuyama, T., \&Onglatco, M. U. (1987). Effects of goals and feedback on performance in groups. Journal of Applied Psychology, 72(3), 407

[42] Mok, S. K. (2016, August 5). [Senior Manager, Department of Engineering Design, ASUS, Interview by author].

[43] Moliner, B., Fuentes, M., \& Gil, I. (2014). Value antecendents in relationship between tourism companies. Journal of Business \& Industrial Marketing, 29(3), 4-4.

[44] Netemeyer, R. G., Boles, J. S., McKee, D. O., \&McMurrian, R. (1997). An investigation into the antecedents of organizational citizenship behaviors in a personal selling context. The Journal of Marketing, 85-98.

[45] Pai, H. C. (2016, August 12). [Senior Manager, Department of Engineering Design, Liteon, Interview by author]

[46] Pang, F. H. (2016, August 21). [Manager, Products R\&D Division, Tatung, Interview by author].

[47] Pirola $\square$ Merlo, A., \& Mann, L. (2004). The relationship between individual creativity and team creativity: Aggregating across people and time. Journal of Organizational Behavior, 25(2), 235-257.

[48] Pisarski, A., Lawrence, S. A., Bohle, P., \& Brook, C. (2008). Organizational influences on the work life conflict and health of shiftworkers. Applied Ergonomics, 39(5), 580-588.

[49] Punj, G., \& Stewart, D. (1983). Cluster analysis in marketing research: Review and suggestions for application. JMR, Journal of Marketing Research (pre-1986), 20(000002), 134-134.

[50] Rahim, A., \&Bonoma, T. V. (1979). Managing organizational conflict: A model for diagnosis and intervention. Psychological reports, 44(3c), 1323-1344.

[51] Robbins, S. P., \& Judge, T. (2003). Essentials of organizational behavior (Vol. 200): Prentice Hall Upper Saddle River.

[52] Robbins, T. L., \&Fredendall, L. D. (2001). Correlates of team success in higher education. The Journal of social psychology, 14l(1), 135-136.

[53] Saavedra, R., Earley, P. C., \& Van Dyne, L. (1993). Complex interdependence in task-performing groups. Journal of Applied Psychology, 78(1), 61 . 
[54] Schmidt, S. M., \&Kochan, T. A. (1972). Conflict: Toward conceptual clarity. Administrative science quarterly, 359-370.

[55] Segi, S. (2013). The Making of Environmental Subjectivity in Managing Marine Protected Areas: A Case Study from Southeast Cebu. Human Organization, 72(4), 336-346.

[56] Simons, T. L., \& Peterson, R. S. (2000). Task conflict and relationship conflict in top management teams: the pivotal role of intragroup trust. Journal of Applied Psychology, 85(1), 102.

[57] Song, L. S. (2016, July 30). [Manager, R\&D Department, Mitac, Interview by author].

[58] Tao, C. F. (2016, July 15). [Director, New Products Development Division, Toongin, Interview by author].

[59] Teng, W. S. (2016, July 13). [Manager, Department of Engineering Design, Behavior, Interview by author].

[60] Tjosvold, D. (1991). The conflict-positive organization: Stimulate diversity and create unity (Vol. 51485): Addison-Wesley.

[61] Tjosvold, D. (1998). Cooperative and competitive goal approach to conflict: Accomplishments and challenges. Applied Psychology, 47(3), 285-313.

[62] Tjosvold, D. (2008). The conflict $\square$ positive organization: It depends upon us. Journal of Organizational Behavior, 29(1), 19-28.

[63] Tsai, C. C. (2016, July 24). [R\&D Director, R\&D Department, Importek, Interview by author].

[64] Tung, S. L. (2016, September 10). [Vice President, Department of R\&D, Portoti, Interview by author].

[65] Turner, A. N., \& Lawrence, P. R. (1965). Industrial jobs and the worker: Harvard Univ.

[66] Ulrich, K., \&Eppinger, S. (2015). Product Design and Development: McGraw-Hill Education.

[67] Van der Vegt, G., \& Van de Vliert, E. (2002). Intragroup interdependence and effectiveness: Review and proposed directions for theory and practice. Journal of managerial psychology, 17(1), 50-67.

[68] Wageman, R. (1995). Interdependence and group effectiveness. Administrative science quarterly, 145-180.

[69] Wong, B. T. (2016, July 22). [Manager, R\&D Department, Transcend, Interview by author].

[70] Wong, D. H. (2016, August 27). [Manager, Department of Mechanic Design, Meiloon, Interview by author].

[71] Yang, C. M. (2016, September, 28). [R\&D Chief, Technology Officer, S\&T, Interview by author]. 